\title{
Research Article \\ Classification of Crystallographic Data Using Canonical Correlation Analysis
}

\author{
M. Ladisa, ${ }^{1}$ A. Lamura, ${ }^{2}$ and T. Laudadio ${ }^{2}$ \\ ${ }^{1}$ Istituto di Cristallografia (IC), CNR, Via Amendola 122/O, 70126 Bari, Italy \\ ${ }^{2}$ Istituto Applicazioni Calcolo (IAC), CNR, Via Amendola 122/D, 70126 Bari, Italy
}

Received 28 September 2006; Revised 10 January 2007; Accepted 4 March 2007

Recommended by Sabine Van Huffel

A reliable and automatic method is applied to crystallographic data for tissue typing. The technique is based on canonical correlation analysis, a statistical method which makes use of the spectral-spatial information characterizing X-ray diffraction data measured from bone samples with implanted tissues. The performance has been compared with a standard crystallographic technique in terms of accuracy and automation. The proposed approach is able to provide reliable tissue classification with a direct tissue visualization without requiring any user interaction.

Copyright () 2007 M. Ladisa et al. This is an open access article distributed under the Creative Commons Attribution License, which permits unrestricted use, distribution, and reproduction in any medium, provided the original work is properly cited.

\section{INTRODUCTION}

One of the main goals of tissue engineering is the reconstruction of highly damaged bony segments. To this aim, it is possible to exploit the patient's own cells, which are isolated, expanded in vitro, loaded onto a bioceramic scaffold, and, finally, reimplanted into the lesion site. Generally, bone marrow stromal cells (BMSC) are adopted, as described in [1]. In this respect it would be important to characterize the structure of the engineered bone and to evaluate whether the BMSC extracellular matrix deposition on a bioceramic scaffold repeats the morphogenesis of the natural bone development. In addition, it is also interesting to look into the interaction between the newly deposited bone and the scaffold in order to recuperate damaged tissues. This is due to the fact that the spatial organization of the new bone and the bonebiomaterial integration is regulated by the chemistry and the geometry of the scaffold used to place BMSC in the lesion site [1-3].

In this context the standard crystallographic approach to detect the different tissues is based on a quantitative analysis performed by the Rietveld technique $[4,5]$. This method allows to determine the relative amounts of different tissue components but it is rather sophisticated and computationally demanding. The aim of this paper is to propose a new technique based on a statistical method called canonical correlation analysis (CCA) [6]. This method is the multivariate variant of the ordinary correlation analysis (OCA) and has already been successfully applied to several applications in biomedical signal processing $[7,8]$. Here, CCA is applied to X-ray diffraction data in order to construct a nosologic image [9] of the bone sample in which all the detected tissues are visualized. The goal is achieved by combining the spectral-spatial information provided by the X-ray diffraction patterns and a signal subspace that models the spectrum of a characteristic tissue type. Such images can be easily interpreted by crystallographers. The paper is organized as follows. In Section 2, we present the mathematical aspects of the CCA method. Then the application of CCA to crystallographic data is reported in Section 3. In Section 4, the numerical results are described and discussed and, finally, we draw our conclusions.

\section{CCA}

CCA is a statistical technique developed by Hotelling in 1936 in order to assess the relationship between two sets of variables [6]. It is a multichannel generalization of OCA, which quantifies the relationship between two random variables $x$ and $y$ by means of the so-called correlation coefficient

$$
\rho=\frac{\operatorname{Cov}[x, y]}{\sqrt{V[x] V[y]}},
$$

where Cov and $V$ stand for covariance and variance, respectively. The correlation coefficient is a scalar with value 
between -1 and 1 that measures the degree of linear dependence between $x$ and $y$. For zero-mean variables, (1) is replaced by

$$
\rho=\frac{E[x y]}{\sqrt{E\left[x^{2}\right] E\left[y^{2}\right]}},
$$

where $E$ stands for expected value. Canonical correlation analysis can be applied to multichannel signal processing as follows: consider two zero-mean multivariate random vectors $\mathbf{x}=\left[x_{1}(t), \ldots, x_{m}(t)\right]^{T}$ and $\mathbf{y}=\left[y_{1}(t), \ldots, y_{n}(t)\right]^{T}$, with $t=1, \ldots, N$, where the superscript $T$ denotes the transpose. The following linear combinations of the components in $\mathbf{x}$ and $\mathbf{y}$ are defined, which, respectively, represent two new scalar random variables $X$ and $Y$ :

$$
\begin{aligned}
& X=w_{x_{1}} x_{1}+\cdots+w_{x_{m}} x_{m}=\mathbf{w}_{x}^{T} \mathbf{x}, \\
& Y=w_{y_{1}} y_{1}+\cdots+w_{y_{n}} y_{n}=\mathbf{w}_{y}^{T} \mathbf{y} .
\end{aligned}
$$

CCA computes the linear combination coefficients $\mathbf{w}_{x}=$ $\left[w_{x_{1}}, \ldots, w_{x_{m}}\right]^{T}$ and $\mathbf{w}_{y}=\left[w_{y_{1}}, \ldots, w_{y_{n}}\right]^{T}$, called regression weights, so that the correlation between the new variables $X$ and $Y$ is maximum. The solution $\mathbf{w}_{x}=\mathbf{w}_{y}=\mathbf{0}$ is not allowed and the new variables $X$ and $Y$ are called canonical variates.

Several implementations of CCA are available in the literature. However, as shown in [7], the most reliable and fastest implementation is based on the interpretation of CCA in terms of principal angles between linear subspaces $[6,10]$. For further details the reader is referred to [7] and references therein. Here, an outline of the aforementioned implementation is provided for the sake of clarity.

\subsection{Algorithm CCA (CCA by computing principal angles)}

Given the zero-mean multivariate random vectors $\mathbf{x}=$ $\left[x_{1}(t), \ldots, x_{m}(t)\right]$ and $\mathbf{y}=\left[y_{1}(t), \ldots, y_{n}(t)\right]$, with $t=$ $1, \ldots, N$.

Step 1. Consider the matrices $\tilde{\mathbf{X}}$ and $\tilde{\mathbf{Y}}$, defined as follows:

$\tilde{\mathbf{X}}=\left[\begin{array}{ccc}x_{1}(1) & \cdots & x_{m}(1) \\ \vdots & & \vdots \\ x_{1}(N) & \cdots & x_{m}(N)\end{array}\right], \quad \tilde{\mathbf{Y}}=\left[\begin{array}{ccc}y_{1}(1) & \cdots & y_{n}(1) \\ \vdots & & \vdots \\ y_{1}(N) & \cdots & y_{n}(N)\end{array}\right]$.

Step 2. Compute the QR decompositions [11] of $\widetilde{\mathbf{X}}$ and $\tilde{\mathbf{Y}}$ :

$$
\begin{aligned}
\tilde{\mathbf{X}} & =\mathbf{Q}_{\tilde{X}} \mathbf{R}_{\tilde{X}}, \\
\tilde{\mathbf{Y}} & =\mathbf{Q}_{\tilde{Y}} \mathbf{R}_{\tilde{Y}},
\end{aligned}
$$

where $\mathbf{Q}_{\tilde{X}}$ and $\mathbf{Q}_{\tilde{Y}}$ are orthogonal matrices and $\mathbf{R}_{\tilde{X}}$ and $\mathbf{R}_{\tilde{Y}}$ are upper triangular matrices.

Step 3. Compute the SVD [11] of $\mathbf{Q}_{\widetilde{X}}^{T} \mathbf{Q}_{\tilde{Y}}$ :

$$
\mathbf{Q}_{\tilde{X}}^{T} \mathbf{Q}_{\tilde{Y}}=\mathbf{U S V}^{T},
$$

where $\mathbf{S}$ is a diagonal matrix and $\mathbf{U}$ and $\mathbf{V}$ are orthogonal matrices. The cosines of the principal angles are given by the diagonal elements of $\mathbf{S}$.

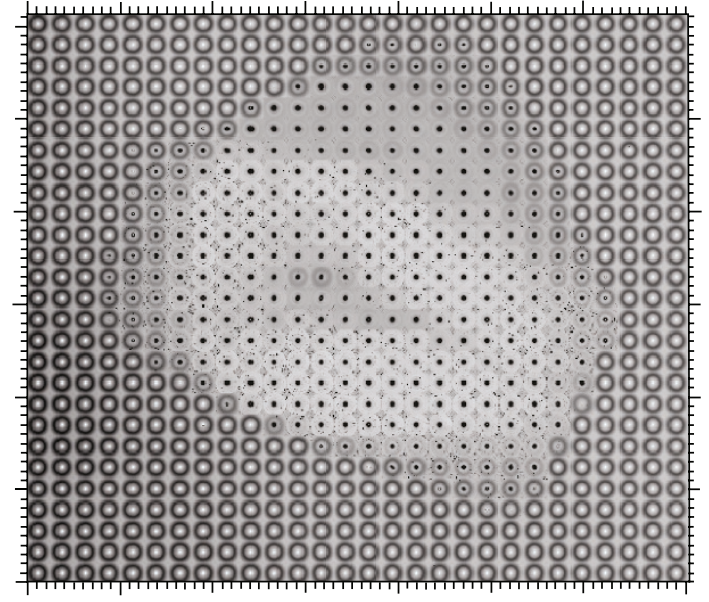

FIGURE 1: X-ray diffraction patterns of the investigated bone sample.

Step 4. Set the canonical correlation coefficients equal to the diagonal elements of the matrix $\mathbf{S}$ and compute the corresponding regression weights as $\mathbf{w}_{\tilde{X}}=\mathbf{R}_{\tilde{X}}^{-1} \mathbf{U}$ and $\mathbf{w}_{\tilde{Y}}=\mathbf{R}_{\tilde{Y}}^{-1} \mathbf{V}$.

The computation of the principal angles yields the most robust implementation of CCA, since it is able to provide reliable results even when the matrices $\tilde{\mathbf{X}}$ and $\tilde{\mathbf{Y}}$ are singular.

\section{CCA APPLIED TO CRYSTALLOGRAPHIC DATA}

During the data acquisition procedure, a number of microscopic X-ray diffraction images (XRDI) displaying the spatial variation of different structural features are acquired. They allow to map the mineralization intensity and bone orientation degree around the pore. The results refer to two different scaffolds with different composition and morphology for two different implantation times. In all the cases the results are similar with respect to the organization of the mineral crystals and collagen micro-fibrils. Thus, for the sake of simplicity, we focus only on one set of such images. Each image represents a two-dimensional X-ray diffraction pattern of a scaffold volume element, called voxel. From the twodimensional diffraction images of the grid in Figure 1 unidimensional signals were obtained by using the algorithm developed in $[12,13]$, each characterized by a 29 scattering angle signal of length $N$. In the proposed tissue segmentation approach, the aim is to detect those voxels whose intensity spectra correlate best with model tissue spectra, which are defined a priori. When applying correlation analysis to XRDI data, the variables $\mathbf{x}$ and $\mathbf{y}$ need to be specified. In OCA $\mathbf{x}$ and $\mathbf{y}$ are univariate variables and, specifically, the $\mathbf{x}$ variable consists of the intensity spectrum of the measured signal contained in each voxel, while the $\mathbf{y}$ variable consists of the model tissue intensity spectrum. The correlation coefficient between $\mathbf{x}$ and $\mathbf{y}$ is computed and assigned to the voxel under investigation. Once each voxel has been processed, a new grid, of the same size of the original set of images, is obtained, 


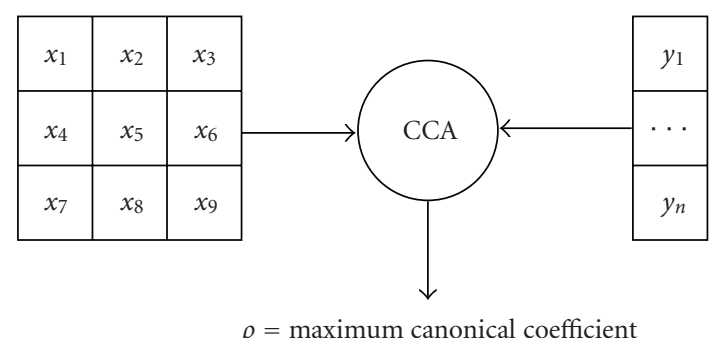

Figure 2: CCA applied to a $3 \times 3$ region of voxels in the diffraction data of Figure 1 and a set of $n$ spectral basis functions [14].

which contains correlation coefficients instead of XRDI signals. This new grid is called correlation map.

The difference between OCA and CCA mainly consists in a different choice of the variables $\mathbf{x}$ and $\mathbf{y}$. In fact, in order to compute the correlation maps, it is possible to exploit the spatial information characterizing the XRDI data set. The variable $\mathbf{x}$ is a multivariate vector with components representing both the intensity spectrum of the considered voxel and the intensity spectra of the neighbor voxels. Several spatial models can be adopted for choosing the neighbor voxels, typical examples of which are described in $[7,8]$. The variable $\mathbf{y}$ also consists of a multivariate vector. Its components represent the basis functions of a signal subspace, that models the characteristic tissue intensity spectrum we are looking for and its possible variations due to Poisson noise that normally affects realistic XRDI data. Several approaches can be adopted in order to model a proper signal subspace; an exhaustive overview is given in [8]. Once the $\mathbf{x}$ and $\mathbf{y}$ variables have been defined, CCA is applied voxel by voxel and the largest canonical coefficient is assigned to the voxel under investigation, so that a correlation map is obtained as in the OCA case. Figure 2 schematically shows the CCA approach when processing a $3 \times 3$ voxel region containing the intensity spectrum $x_{5}$ along with its neighbor intensity spectra [14]. In this particular special model, called the " $3 \times 3$ " model, the variable $\mathbf{x}$ contains 9 components, namely, $\mathbf{x}=\left[\mathbf{x}_{1}, \ldots, \mathbf{x}_{9}\right]^{T}$.

\subsection{Choice of the spatial model}

As already mentioned in the previous section, several spatial models can be chosen when applying CCA. As a particular case, OCA can be considered as a single-voxel model. The performance of the following spatial models [8] was investigated:

(i) the single-voxel model (OCA);

(ii) the $3 \times 3$ model $(3 \times 3)$ :

$$
\mathbf{x}=\left[\mathbf{x}_{1}, \ldots, \mathbf{x}_{9}\right]^{T}
$$

(iii) the $3 \times 3$ model without corner voxels:

$$
\mathbf{x}=\left[\mathbf{x}_{2}, \mathbf{x}_{4}, \mathbf{x}_{5}, \mathbf{x}_{6}, \mathbf{x}_{8}\right]^{T}
$$

(iv) the symmetric $3 \times 3$ model:

$$
\mathbf{x}=\left[\mathbf{x}_{5}, \frac{\mathbf{x}_{1}+\mathbf{x}_{9}}{2}, \frac{\mathbf{x}_{2}+\mathbf{x}_{8}}{2}, \frac{\mathbf{x}_{3}+\mathbf{x}_{7}}{2}, \frac{\mathbf{x}_{4}+\mathbf{x}_{6}}{2}\right]^{T} ;
$$

(v) the symmetric $3 \times 3$ model without corner voxels $(\mathrm{s} 3 \times 3 \mathrm{wcv})$ :

$$
\mathbf{x}=\left[\mathbf{x}_{5}, \frac{\mathbf{x}_{2}+\mathbf{x}_{8}}{2}, \frac{\mathbf{x}_{4}+\mathbf{x}_{6}}{2}\right]^{T}
$$

(vi) the symmetric filter (sf):

$$
\mathbf{x}=\left[\mathbf{x}_{5}, \frac{\mathbf{x}_{2}+\mathbf{x}_{4}+\mathbf{x}_{6}+\mathbf{x}_{8}}{4}\right]^{T}
$$

(vii) the constrained symmetric filter (constrained sf): for the sake of completeness we also consider a constrained version of the previous spatial model, where the weights in the vector $\mathbf{w}_{x}$ are constrained to be nonnegative [15]. The constrained solution ensures that sufficient weight is put on the center voxel so as to avoid a possible interference from surrounding voxels. To this end, the spatial model is chosen as

$$
\mathbf{x}=\left[\mathbf{x}_{5}, \mathbf{x}_{5}+\frac{\mathbf{x}_{2}+\mathbf{x}_{4}+\mathbf{x}_{6}+\mathbf{x}_{8}}{4}\right]^{T} .
$$

The optimal constrained CCA solution is then found by applying CCA as follows.

(1) Apply CCA with

$$
\mathbf{x}=\left[\mathbf{x}_{5}, \mathbf{x}_{5}+\frac{\mathbf{x}_{2}+\mathbf{x}_{4}+\mathbf{x}_{6}+\mathbf{x}_{8}}{4}\right]^{T} .
$$

If the weights in $\mathbf{w}_{x}$ are all positive (or all negative), this is the solution to the constrained problem, otherwise apply (2).

(2) Apply twice CCA with, respectively,

$$
\begin{gathered}
\mathbf{x}=\left[\mathbf{x}_{5}\right]^{T}, \\
\mathbf{x}=\left[\mathbf{x}_{5}+\frac{\mathbf{x}_{2}+\mathbf{x}_{4}+\mathbf{x}_{6}+\mathbf{x}_{8}}{4}\right]^{T} .
\end{gathered}
$$

The CCA approach providing the highest canonical correlation coefficient gives the solution to the constrained problem.

The results are described in the numerical results section.

\subsection{Choice of the subspace model}

Concerning the choice of the $y$ variable, the so-called Taylor model $[8,16]$ was considered in order to define the proper signal subspace able to model the characteristic tissue spectra and their possible variations. In our application, five subspace models were defined. More precisely, in order to define the first component of the variable $y$, five intensity 


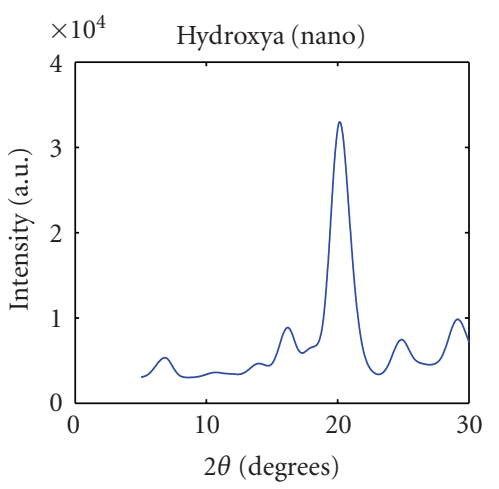

(a)

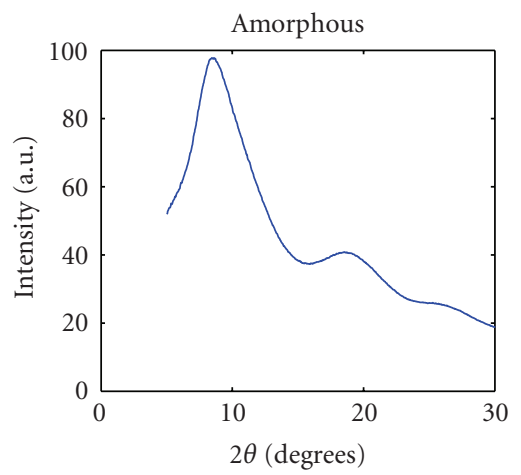

(b)

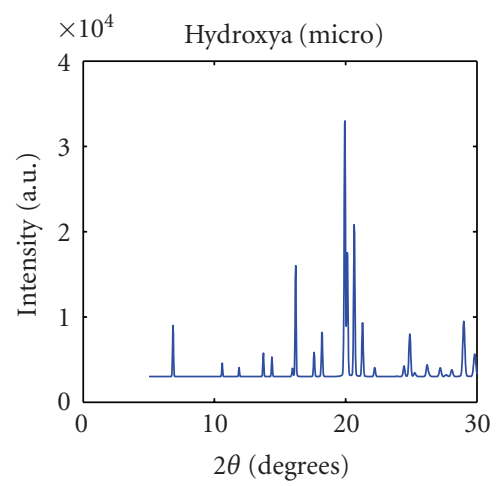

(c)

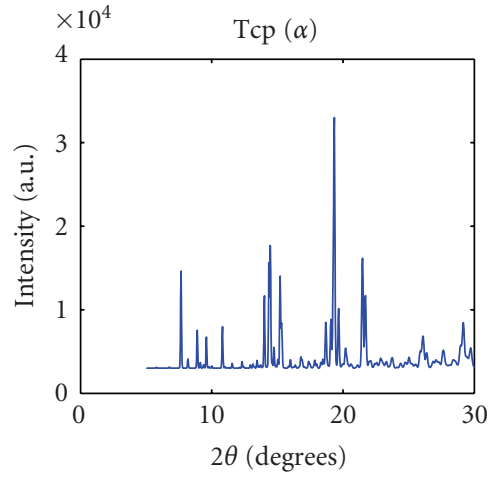

(d)

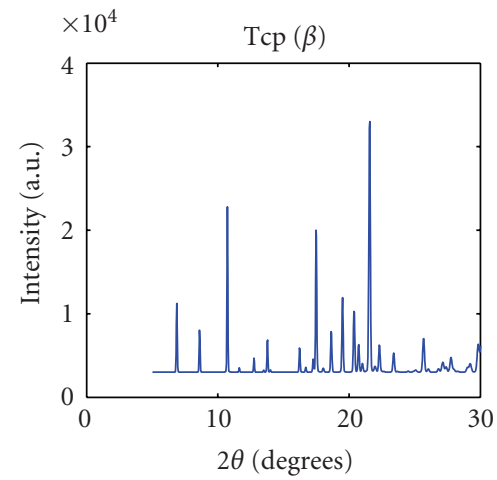

(e)

FIGURE 3: Intensity spectra of characteristic tissue signals.

spectra were selected as characteristic of the component tissues: silicon-stabilized tricalcium phosphate (Tcp), with two different compositions named $\alpha$ and $\beta$, hydroxyapatite ( $\mathrm{Ha}$ ), with two different crystal sizes at micrometer and nanometer scale, and the amorphous tissue representing the old natural bone. These intensity spectra represent our models and will be called profile models. The first component of the $\mathbf{y}$ variable was then defined as the chosen profile model. The second component of $\mathbf{y}$ was obtained as the first-order derivative of the first component, approximated by first-order finite differences. For the sake of clarity, the procedure to compute the aforementioned subspace model is here outlined.

\section{The Taylor subspace model}

Step 1. Choose the profile model $P(n), n=1, \ldots, N$, where $N=1024$, corresponding to the considered tissue type.

Step 2. Set the components of the variable $y$ as

$$
\begin{gathered}
y_{1}(n)=P(n), \\
y_{2}(n)=\frac{P(n+1)-P(n)}{\Delta \theta},
\end{gathered}
$$

where $n=1, \ldots, 1024$ and $\Delta \theta=0.024^{\circ}$ is the sampling angle.
Such a subspace model accounts for possible frequency shifts of the peaks. Indeed, a simultaneous peak shift may occur in experimental spectra due to an instrumental bias (e.g., zero-angle shift). This shift is negligible for the broad spectra of hydroxya (nano) and amorphous, while it may affect the other spectra (see Figure 3). Finally, for the single voxel approach, only one component was considered and set equal to the first component of the Taylor subspace model, namely, $\mathbf{y}=\mathbf{y}_{1}$.

\section{NUMERICAL RESULTS}

In the experiment MD67, carried out at the European Synchrotron Radiation Facility (ESRF) ID13 beamline, a local interaction between the newly formed mineral crystals in the engineered bone and the biomaterial has been investigated by means of microdiffraction with submicron spatial resolution. Combining wide angle X-ray scattering (WAXS) and small angle X-ray scattering (SAXS) with high spatial resolution determines the orientation of the crystallographic geometry inside the bone grains and the orientation of the mineral crystals and collagen micro-fibrils with respect to the scaffold. In [1-3] a quantitative analysis of both the SAXS and WAXS patterns was performed showing that the grain size is compatible with a model for mineralization in early stage. In particular, the performance of Skelite ${ }^{T M}$ (Millenium Biologix 


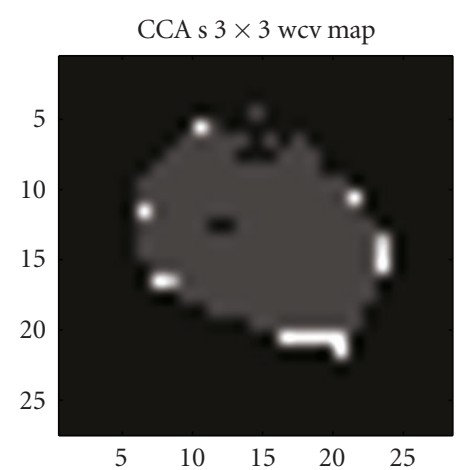

(a)

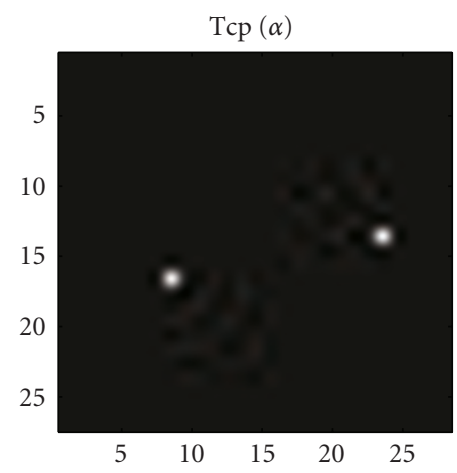

(d)

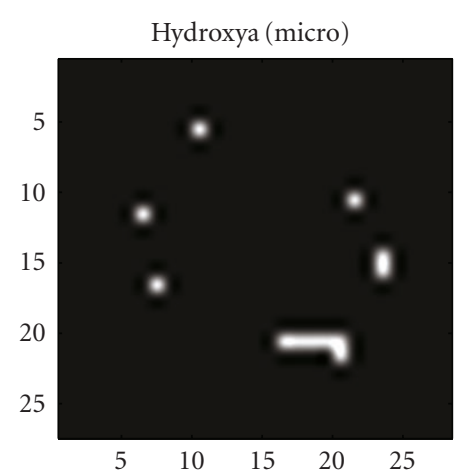

(b)

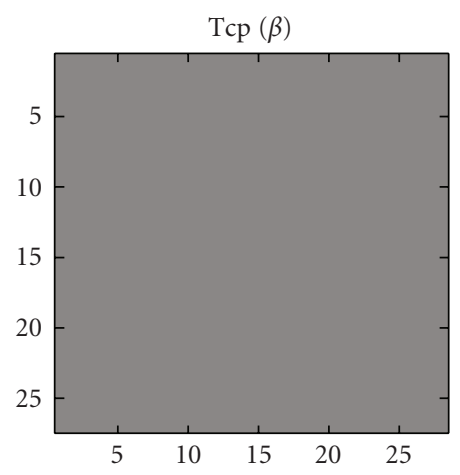

(e)

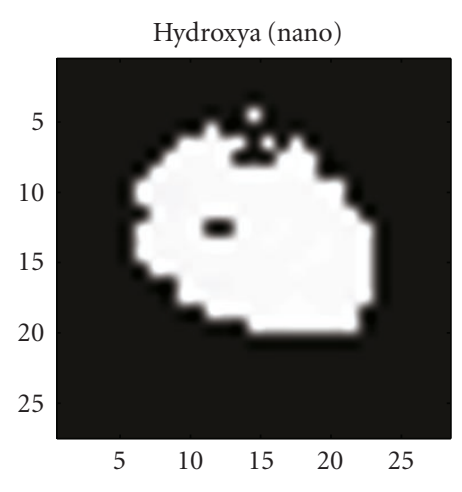

(c)

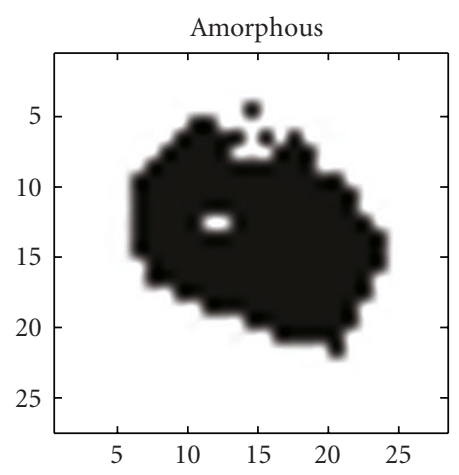

(f)

FIgURE 4: The nosologic image and the correlation maps (white corresponds to highest canonical coefficient) obtained from diffraction data of Figure 1 by CCA s $3 \times 3$ wcv (black: amorphous, dark gray: hydroxya (nano), gray: $\operatorname{Tcp}(\alpha)$, light gray: hydroxya (micro)).

Corp., Kingston, Canada), a clinically available scaffold based on Ha and Tcp, has been evaluated.

Figure 1 shows a screenshot of several microscopic XRDI displaying the spatial variation of different structural features, thus allowing to map the mineralization intensity and bone orientation degree around the scaffold pore. The results obtained by applying the standard quantitative analysis [5] suggest that the ratio $\mathrm{Tcp} / \mathrm{Ha}$ could change in proximity of the interface scaffold/new bone and with the implantation time. CCA was then used in order to retrieve the possible material types characterizing the sample under investigation.

As already specified in Section 3.2, five different y variables were defined, one for each tissue. CCA was applied between the experimental unidimensional data set and the above mentioned $\mathbf{y}$ variables. We obtained five different correlation maps and comparing, pixel by pixel, the five canonical correlation coefficients, we built the nosologic image by assigning the considered pixel to the tissue with the highest canonical coefficient. We tested all the different spatial models reported in Section 3 and the best performance was obtained by using the symmetric $3 \times 3$ model without corner voxels and the symmetric filter model. The reason of such a behavior is due to the choice of the neighbors to be used.
Specifically the single voxel approach does not exploit any spatial information which makes the tissue detection more reliable. With respect to the models involving information from neighbors, we might expect that only the nearest ones should be relevant. This is because the diffusion of materials does not occur on space scales larger than $1 \mu \mathrm{m}$ within the time scale of the experiment, which is the spatial sampling distance in diffraction measurements.

In Figure 4 the correlation maps obtained by CCA s $3 \times 3$ wcv are visualized for amorphous, Ha (nano), Ha (micro), $\operatorname{Tcp}(\alpha)$, and Tcp $(\beta)$. The first frame in Figure 4 shows the nosologic image, where the gray tones denote the tissue types as follows: the black denotes the amorphous, the dark gray indicates the $\mathrm{Ha}$ (nano), the gray corresponds to $\operatorname{Tcp}(\alpha)$, the light gray denotes $\mathrm{Ha}$ (micro).

In Figure 5, the nosologic images obtained by CCA when applying different spatial models are shown. As it can be observed, the symmetric $3 \times 3$ model without corner voxels gives a nosologic image that resembles the pattern of Figure 1 quite well, so does the CCA sf spatial model of Figure 5. This is because, as already argued, both of them exploit the information from the nearest neighbors. However, the comparison with the two-dimensional X-ray patterns of 


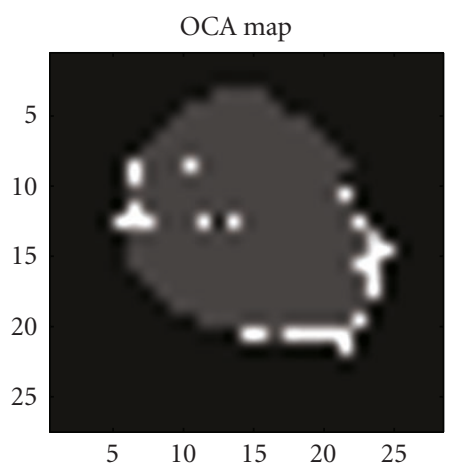

(a)

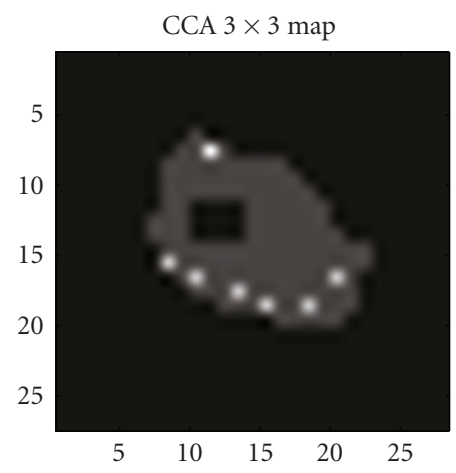

(b)

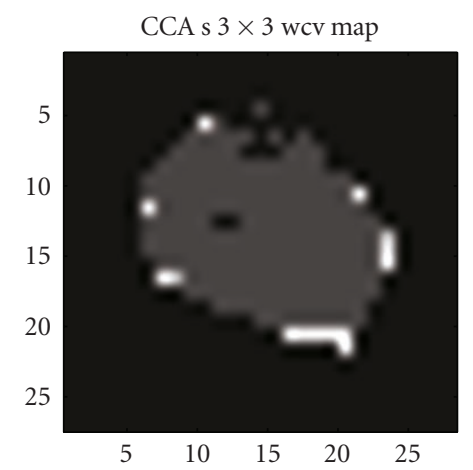

(c)

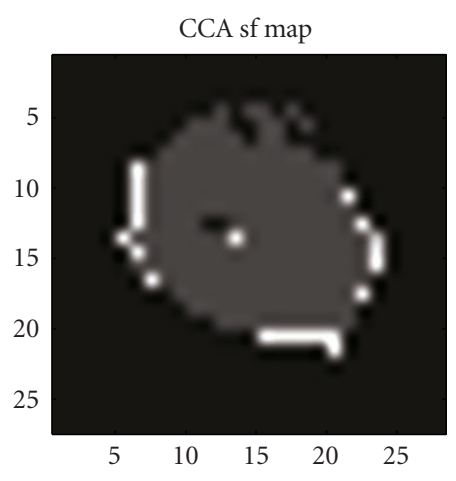

(d)

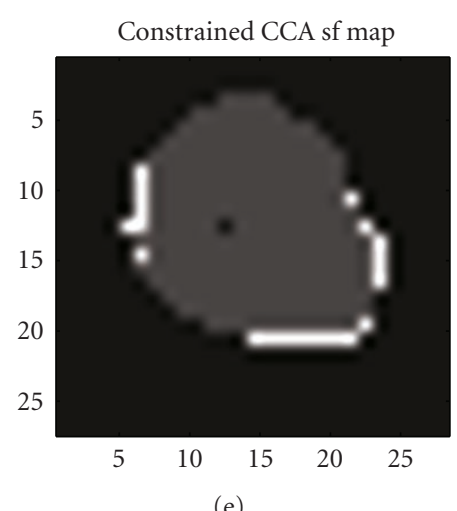

(e)

FIgUre 5: Nosologic images obtained from diffraction data of Figure 1 by CCA when applying different spatial models (see the text for details).

Figure 1 is just a guide to the eye since CCA is applied to onedimensional X-ray patterns, as previously stated. The latter profiles show details that cannot be quantitatively appreciated by a simple visual inspection of Figure 1 . We made a comparison with a quantitative analysis performed by the Rietveld technique. This is a standard way to investigate the unidimensional powder pattern [5] and to determine the relative amounts of different components. We decided to consider one-dimensional cross-sections of Figure 1. For instance, in top parts of Figures 6-7 we report two arbitrary cross-sections cut across and along the sample (similar results were obtained by considering different cross sections), respectively. In both figures, we also show the results of CCA analysis (middle parts) obtained by the different spatial models of Figure 5, and standard quantitative analysis (bottom parts). We can see that the symmetric $3 \times 3$ model without corner voxels and the symmetric filter are helpful in spotting the expected tissue component in each scattering voxel. It is worth to stress that the amorphous contribution, according to the quantitative analysis, is largely dominant, as it can be seen in bottom plots of Figures 6-7, and this may sometimes produce a misassignment at interfaces with other tissues as, for instance, it happens for the 5th pixel from left in CCA s $3 \times 3 \mathrm{wcv}$ in Figure 6 . This is due to the fact that in the considered multivoxel approach the neighbor pixels are equally weighted and do not account for the overwhelming contribution of the amorphous. In Figure 7, the classification done by
CCA s $3 \times 3 \mathrm{wcv}$ looks the best when compared to the results of the quantitative analysis. Similar results are obtained by using other cross-sections. According to the standard analysis, it is evident that the lower is the amorphous signal along the cross section, the higher is the hydroxya (nano) signal. Therefore, the crystallographic approach requires a further inspection by the user in order to assign the prevailing tissue to the considered voxel. On the contrary, CCA is sensitive to the subleading signal and is able to assign it to the considered pixel without any further user interaction.

\section{CONCLUSIONS}

In this paper, we have proposed a method for tissue typing of XRDI data acquired from a clinically available scaffold implanted in a damaged bone. The technique is based on canonical correlation analysis and is able to provide reliable tissue classification with its direct visualization and without requiring any user interaction. These results give important indication about the resorption mechanism and the role of stromal cells in the structural change of scaffold. Canonical correlation analysis reveals as a valid tool for a systematic analysis of the materials as they appear in the X-ray diffraction patterns. Probing to what extent, this new statistical technique provides convincing results as to other features of the sample, for instance, the mineralization orientation is an important endeavour to be investigated in the future. 


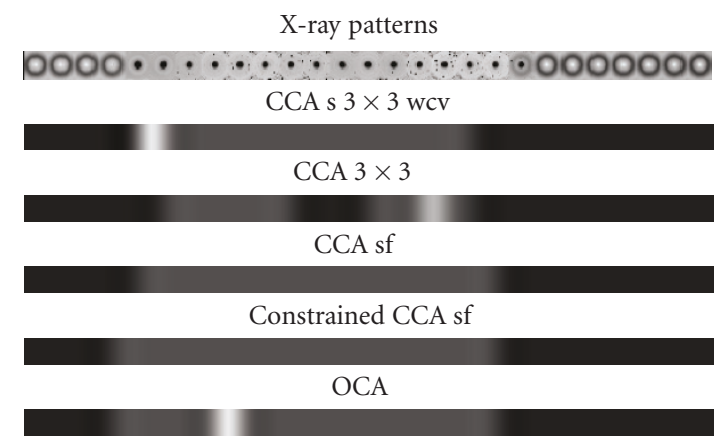

Quantitative crystallographic analysis

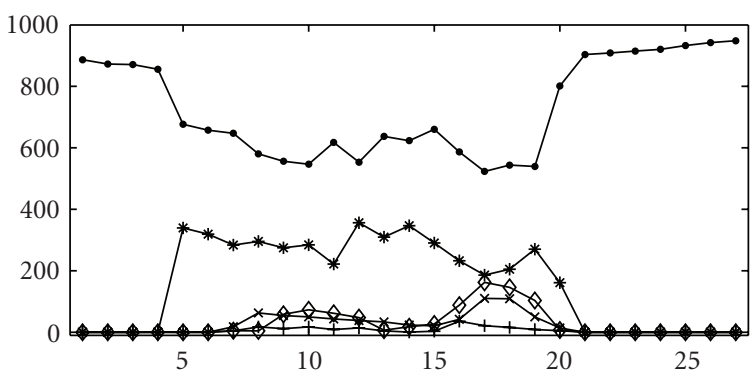

Figure 6: Top: cross-section from top to bottom of Figure 1 along the 11th column from left. Middle: the corresponding crosssections of the different CCA maps of Figure 5 (black: amorphous, light gray: hydroxya (micro), gray: Tcp $(\alpha)$, dark gray: hydroxya (nano)). Bottom: quantitative crystallographic analysis $(\bullet$ : amorphous, $*$ : hydroxya (nano), $\diamond: \operatorname{Tcp}(\alpha), \times$ : hydroxya (micro), +: $\operatorname{Tcp}(\beta))$.

X-ray patterns

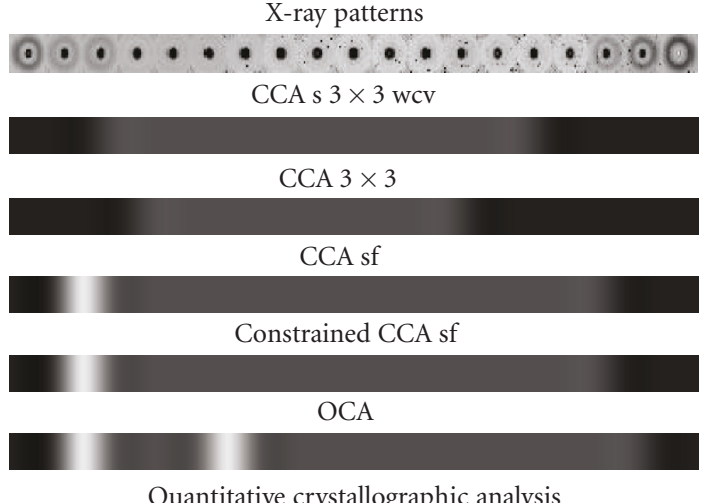

Quantitative crystallographic analysis

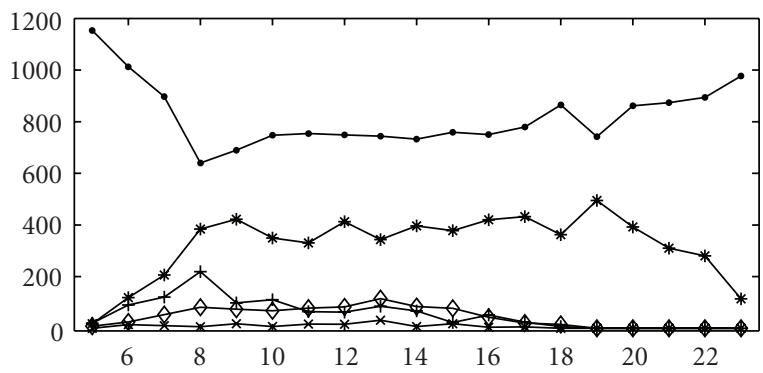

Figure 7: Top: cross-section from left to right of Figure 1 along the 9th row from top. Middle: the corresponding cross-sections of the different CCA maps of Figure 5 (black: amorphous, light gray: hydroxya (micro), dark gray: hydroxya (nano)). Bottom: quantitative crystallographic analysis $(\bullet$ : amorphous, $*$ : hydroxya (nano), $\diamond$ : hydroxya (micro), $\times: \operatorname{Tcp}(\beta),+: \operatorname{Tcp}(\alpha))$.

\section{ACKNOWLEDGMENTS}

The authors thank A. Cedola, A. Cervellino, C. Giannini, and A. Guagliardi for kindly providing them with experimental crystallographic data and quantitative analysis reported in Figures 6-7.

\section{REFERENCES}

[1] A. Cedola, M. Mastrogiacomo, M. Burghammer, et al., "Structural study with advanced X-ray microdiffraction technique of bone regenerated by bone marrow stromal cells," Physics in Medicine and Biology, vol. 51, no. 6, pp. N109-N116, 2006.

[2] A. Cedola, M. Mastrogiacomo, S. Lagomarsino, et al., "X-ray diffraction imaging applied to in vivo bone engineering," to appear in Spectrochimica Acta B.

[3] V. Komlev, F. Peyrin, M. Mastrogiacomo, et al., "3D analysis of scaffold material and bone growth into tissue engineering constructs via X-ray computed microtomography," submitted to Tissue Engineering.

[4] R. A. Young, The Rietveld Method, Oxford University Press, New York, NY, USA, reprint edition, 1993.

[5] A. Guinier, X-Ray Diffraction in Crystals, Imperfect Crystals, and Amorphous Bodies, Dover, New York, NY, USA, reprint edition, 1994.

[6] H. Hotelling, "Relation between two sets of variates," Biometrika, vol. 28, pp. 321-377, 1936.

[7] T. Laudadio, P. Pels, L. De Lathauwer, P. Van Hecke, and S. Van Huffel, "Tissue segmentation and classification of MRSI data using canonical correlation analysis," Magnetic Resonance in Medicine, vol. 54, no. 6, pp. 1519-1529, 2005.

[8] O. Friman, Adaptive analysis of functional MRI data, Ph.D. thesis, Department of Biomedical Engineering, Linköping University, Linköping, Sweden, 2003.

[9] F. S. De Edelenyi, C. Rubin, F. Esteve, et al., "A new approach for analyzing proton magnetic resonance spectroscopic images of brain tumors: nosologic images," Nature Medicine, vol. 6, no. 11, pp. 1287-1289, 2000.

[10] $\mathrm{H}$. Zha, The singular value decomposition theory, algorithms and applications, Ph.D. thesis, Pennsylvania State University, Philadelphia, Pa, USA, 1993.

[11] G. H. Golub and C. Van Loan, Matrix Computations, Johns Hopkins University Press, New York, NY, USA, 1996.

[12] A. Cervellino, C. Giannini, A. Guagliardi, and M. Ladisa, "Disentangling instrumental broadening," Journal of Applied Crystallography, vol. 38, no. 4, pp. 685-687, 2005.

[13] A. Cervellino, C. Giannini, A. Guagliardi, and M. Ladisa, "Folding a two-dimensional powder diffraction image into a one-dimensional scan: a new procedure," Journal of Applied Crystallography, vol. 39, no. 5, pp. 745-748, 2006.

[14] O. Friman, J. Cedefamn, P. Lundberg, M. Borga, and H. Knutsson, "Detection of neural activity in functional MRI using canonical correlation analysis," Magnetic Resonance in Medicine, vol. 45, no. 2, pp. 323-330, 2001.

[15] S. Das and P. Sen, "Restricted canonical correlations," Linear Algebra and Its Applications, vol. 210, pp. 29-47, 1994.

[16] K. J. Friston, P. Fletcher, O. Josephs, A. Holmes, M. D. Rugg, and R. Turner, "Event-related fMRI: characterizing differential responses," NeuroImage, vol. 7, no. 1, pp. 30-40, 1998. 
M. Ladisa received the Laurea and Ph.D. degrees in physics from the University of Bari, Bari, Italy, in 1997 and 2001, respectively. He is currently a Researcher with the Istituto di Cristallografia (IC), National Research Council (CNR), Bari, Italy.

A. Lamura received the Laurea and Ph.D. degrees in physics from the University of Bari, Bari, Italy, in 1994 and 2000, respectively. He is currently a Researcher with the Istituto per le Applicaizoni del Calcolo (IAC), National Research Council (CNR), Bari, Italy.

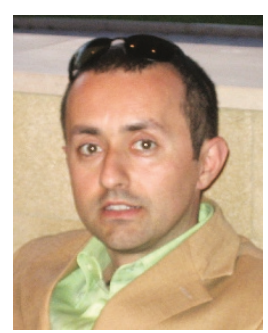

T. Laudadio received the Laurea degree in mathematics from the University of Bari, Bari, Italy, in 1992, and the Ph.D. degree in electrical engineering from the Katholieke Universiteit Leuven, Leuven, Belgium, in 2005. She is currently a Research Fellow with the Istituto di Studi sui Sistemi Intelligenti per l'Automazione (ISSIA), National Research Council (CNR), Bari, Italy.

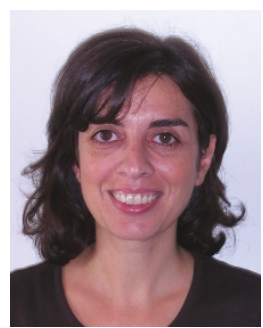

